University of Nebraska - Lincoln

DigitalCommons@University of Nebraska - Lincoln

2-8-2001

\title{
Effect of carrier-gas pressure on barrier to nucleation: Monte Carlo simulation of water/nitrogen system
}

\author{
K.J. Oh \\ University of Nebraska-Lincoln \\ Xiao Cheng Zeng \\ University of Nebraska-Lincoln, xzeng1@unl.edu
}

Follow this and additional works at: https://digitalcommons.unl.edu/chemzeng

Part of the Chemistry Commons

Oh, K.J. and Zeng, Xiao Cheng, "Effect of carrier-gas pressure on barrier to nucleation: Monte Carlo simulation of water/nitrogen system" (2001). Xiao Cheng Zeng Publications. 47.

https://digitalcommons.unl.edu/chemzeng/47

This Article is brought to you for free and open access by the Published Research - Department of Chemistry at DigitalCommons@University of Nebraska - Lincoln. It has been accepted for inclusion in Xiao Cheng Zeng Publications by an authorized administrator of DigitalCommons@University of Nebraska - Lincoln. 


\title{
Effect of carrier-gas pressure on barrier to nucleation: Monte Carlo simulation of water/nitrogen system
}

\author{
K. J. Oh and X. C. Zeng ${ }^{\text {a) }}$ \\ Department of Chemistry, University of Nebraska-Lincoln, Lincoln, Nebraska 68588
}

(Received 18 October 2000; accepted 16 November 2000)

\begin{abstract}
Carrier gases are used in most nucleation experiments for releasing the latent heat generated during vapor condensation. In the analysis of experimental data it is often assumed that the carrier gas is inert and would not participate in the nucleation process of the target gas. Several recent nucleation experiments show that the influence of carrier gases to nucleation rate is not negligible under certain conditions. To gain more insight into the carrier-gas effect, we carry out Monte Carlo simulation to compute the free energy of formation of water clusters in the presence of a nitrogen carrier gas. At fixed temperature $(240 \mathrm{~K})$ and chemical potential, it is found that the barrier height to nucleation increases with the carrier-gas pressure. This barrier enhancement is attributed to the increase of equilibrium vapor pressure of water in the presence of carrier gas, which results in a decrease of supersaturation. It is also found that the simulation results are consistent with the binary-nucleation theorem. (C) 2001 American Institute of Physics. [DOI: 10.1063/1.1339222]
\end{abstract}

\section{INTRODUCTION}

Carrier gases (or background gases) are commonly used as a latent-heat reservoir to maintain an isothermal environment in most vapor-nucleation experiments. If the carrier gases were not present, the latent heat would heat up the condensing clusters and the condensation process would not proceed under the isothermal condition. On analyzing experimental data, it is often assumed that carrier gas is just a spectator and not involved in the nuclei formation of a target gas. Based on this assumption, the conventional theory of unary homogeneous nucleation can be applied without considering the effect of the second component in the gas mixture- the carrier gas. However, the condition under which this assumption is applicable remains an open question. To accurately evaluate the rate of nucleation of target gas, one should examine the extent to which the carrier gas affects the target-gas nucleation under various experimental conditions.

Recently, several nucleation experiments have shown that the influence of carrier gas to rate of nucleation can be appreciable under certain conditions. In diffusion cloudchamber experiments, ${ }^{1-5}$ for example, marked effects of carrier-gas pressure on the rate of nucleation and the critical supersaturation were seen. For a water/nitrogen system, however, a recent experiment ${ }^{6}$ using a high pressure pulseexpansion wave tube demonstrated that when the nucleation rate is plotted as a function of conventional supersaturation (i.e., the ratio of the partial vapor pressure of the supersaturated vapor to the equilibrium vapor pressure of the pure component), the carrier-gas pressure effect is hardly discernible.

In an attempt to explain the carrier-gas effect observed in diffusion cloud-chamber experiments, Fisk and $\mathrm{Katz}^{7}$ examined effects of nonideality of carrier gas and nonzero

a)Electronic mail: xzeng1@unl.edu compressibility of the critical nuclei on the rate of nucleation. They found that none of these effects accounts for the observation of an apparent decrease in nucleation rate with increasing total pressure. Kane and El-Shall ${ }^{8}$ found that the carrier-gas effect observed in diffusion cloud chamber experiments arises from the slowdown of the dynamics of growth of the nuclei into detectable droplets. Reiss and co-workers ${ }^{9-11}$ suggested that the carrier-gas effect may be due to the screening effect of a kinetic origin by which the drop's attractive potential is weakened by encounters of incoming molecules with other vapor or carrier-gas molecules. Luijten et al. ${ }^{6,12,13}$ proposed that the carrier-gas effect can be explained by two competing factors: the enhanced vapor pressure which hampers nucleation and the reduced surface tension which facilitates nucleation. Based on the nucleation theorem for binary nucleation, Oxtoby and Laaksonen ${ }^{14}$ showed that the carrier-gas effect is generally small. A similar conclusion was also drawn by Ford within the framework of the classical nucleation theory. ${ }^{15,16}$ Kashchiev ${ }^{17}$ showed that when the pressure is not much greater than the atmospheric pressure the carrier-gas effect is relatively small but is more pronounced for less supersaturated vapor.

In this paper we present, to our knowledge, the first molecular simulation study of the carrier-gas effect on vapor nucleation. Molecular simulation allows us to gain more insight on the carrier-gas effect at the molecular level. Specifically, we studied the nucleation of a model water in the presence of nitrogen gas. We calculated the free energy of formation of water clusters as a function of nitrogen gas pressure. To this end, we used a small-ensemble Monte Carlo simulation method ${ }^{18,19}$ which is similar to the one originally developed by Kusaka et al. ${ }^{20}$ This Monte Carlo method is particularly useful when applied to highly compact clusters. ${ }^{19,21,22}$ 


\section{THEORETICAL FORMALISM}

We view the supersaturated vapor as a mixture of physical clusters. Each configuration of a supersaturated vapor is characterized by a specific cluster size distribution which satisfies the equation

$$
N=\sum_{i=1}^{i_{\max }} i n_{i},
$$

where $n_{i}$ is the number of clusters with $i$ molecules, and $i_{\max }$ is the upper bound of the cluster size used to maintain the supersaturated vapor in metastable equilibrium. By maximizing the total partition function of the supersaturated vapor (in the framework of physical cluster theory), we obtain the most probable cluster size distribution

$$
n_{i}=q_{i} e^{\beta \mu_{i}\left(p_{i}\right)},
$$

where $\beta=1 / k_{B} T, \mu_{i}\left(p_{i}\right)$ is the chemical potential of the clusters at their partial pressure $p_{i}$, and $q_{i}$ is the cluster partition function. Note that in deriving Eq. (2) we neglect the cluster-vapor and cluster-cluster interactions based on the fact that the vapor density is extremely small. We also obtain the well-known law of mass action

$$
\mu_{i}\left(p_{i}\right)=i \mu_{1}\left(p_{1}\right)
$$

where $\mu_{1}$ is the chemical potential of the monomers in the vapor. We define the free energy of formation $\Delta G_{i}$ of an $i$-mer as

$$
\Delta G_{i}=\mu_{i}\left(p_{1}\right)-\mu_{i}\left(p_{i}\right) .
$$

By employing a simple formula of ideal gas $\mu_{i}\left(p_{1}\right)$ $\approx \mu_{i}\left(p_{i}\right)+k_{B} T \ln n_{1} / n_{i}$, we arrive at the expression

$$
\Delta G_{i}=-k_{B} T \ln \frac{n_{i}}{n_{1}} .
$$

$\Delta G_{i}$ in Eq. (5) is also the Gibbs free-energy change in the association reaction of $i$ monomers to an $i$-mer at constant pressure $p_{1}$. Thus, we can use the standard relation $\Delta G_{i}=-k_{B} T \ln K_{p}$, where $K_{p}$ is the equilibrium constant for the association reaction.

In the computer simulation, Eq. (5) is written as

$$
\Delta G_{i}=-k_{B} T \ln \frac{P_{i}}{P_{1}},
$$

where $P_{i}=n_{i} / M$ and represents the probability of finding $i$-mers in the supersaturated vapor. Here, $M$ acts as the partition function of a statistical ensemble

$$
M=\sum_{i=1}^{i_{\max }} n_{i}=\sum_{i=1}^{i_{\max }} q_{i} e^{\beta \mu_{i}\left(p_{i}\right)}
$$

in which exchange of cluster molecules with the monomers is allowed. $P_{i}$ can be evaluated using an umbrella-sampling simulation method ${ }^{18,19}$ which will be described in the next section.

In the physical cluster theory ${ }^{18}$ the cluster partition function $q_{i}$ can be written as

$$
\begin{aligned}
q_{i}= & \frac{\gamma_{T}^{i} \gamma_{R}^{i}}{i !} \int_{V} H\left(\mathbf{r}_{1}, \ldots, \mathbf{r}_{i}\right) \\
& \times \exp \left(-\beta W_{i}\right) d \mathbf{r}_{1}, \ldots, d \mathbf{r}_{i} d \mathbf{\Omega}_{1}, \ldots, d \mathbf{\Omega}_{i} \\
= & \frac{\gamma_{T}^{i-1} \gamma_{R}^{i} i^{3} V}{i !} \int_{v} H\left(\mathbf{r}_{1}^{\prime}, \ldots, \mathbf{r}_{i-1}^{\prime}\right) \\
& \times \exp \left(-\beta W_{i}\right) d \mathbf{r}_{1}^{\prime}, \ldots, d \mathbf{r}_{i-1}^{\prime} d \mathbf{\Omega}_{1}, \ldots, d \mathbf{\Omega}_{i} \\
= & \frac{\gamma_{T} i^{3} V}{i !} q_{i}^{*},
\end{aligned}
$$

where $V$ is the volume of the supersaturated vapor, $\gamma_{T}$ and $\gamma_{R}$ are the terms related to thermal de Broglie wavelength of water molecules corresponding to translational and rotational motion, respectively, and $q_{i}^{*}$ is the cluster partition function in the center-of-mass coordinate. In Eqs. (8) and (9), $W_{i}$ is the potential energy of cluster, i.e.,

$$
W_{i}=U_{i}+U_{i c}
$$

where $U_{i}$ is the interaction energy among $i$ water molecules and $U_{i c}$ is the interaction energy between water molecules and carrier-gas molecules; $\mathbf{r}$ and $\boldsymbol{\Omega}$ represent translational and rotational coordinates of water molecules, and $\int d \boldsymbol{\Omega}$ $=\boldsymbol{\Omega}=8 \pi^{2}$. The prime in Eq. (9) represents the coordinates with respect to the center of mass of the cluster and the term $V$ comes from the integration over the center-of-mass coordinate of the cluster. The cluster criterion is imposed through a step function $H$, which is 1 if the cluster criterion is met and 0 otherwise. In practice, the integration in Eq. (9) is taken only over a small volume $v$ as long as $v$ is larger than the excluded volume of the cluster. This is because $q_{i}^{*}$ would not be affected by enlarging the range of integration beyond $v$.

The presence of carrier-gas molecules is included implicitly in the term $W_{i}$ in Eq. (11). This means that only the first-order contribution of the carrier-gas molecules to the free energy of formation of water clusters is taken into account. Higher order contributions such as the cluster-cluster interactions mediated by the carrier-gas molecules are neglected because of the extremely low density of vapor and small concentration of clusters compared to that of monomers.

The physical cluster is generally defined in the centerof-mass coordinate system. ${ }^{22}$ Because water molecules are highly associative, water clusters are quite compact. As a result, the center-of-mass fluctuation can be reduced by just fixing an arbitrary water molecule in the cluster. Theoretically, this means that $q_{i}^{*}$ can be also written as

$$
q_{i}^{*} \approx \frac{\gamma_{T}^{i-1} \gamma_{R}^{i}}{i !} \int_{v} \exp \left(-\beta W_{i}\right) d \mathbf{r}_{2}, \ldots, d \mathbf{r}_{i} d \mathbf{\Omega}_{1}, \ldots, d \mathbf{\Omega}_{i}
$$

where the position of an arbitrary molecule in the cluster is fixed, instead of the center of mass of the cluster. This approximation is operationally similar to the one used by 
Kusaka et $a l .{ }^{20}$ in the study of pure water nucleation. Our study indicates that the free energy of formation of water clusters was insensitive to the condition whether the center of mass of the cluster is fixed or one of the molecules in the cluster is fixed.

\section{MONTE CARLO SIMULATION}

In the Monte Carlo simulation we used the SPC/E model of water, ${ }^{23}$ which is a three-site potential model. Only the oxygen site interacts with the other oxygen sites as well as the carrier-gas molecules via the Lennard-Jones (LJ) potential. The nitrogen carrier-gas molecule is modeled as a single-site Lennard-Jones sphere with LJ parameters $\epsilon$ $=0.7608 \mathrm{~kJ} / \mathrm{mol}$ and $\sigma=3.68 \AA$. The LJ mixing parameters between the oxygen site and nitrogen molecule are given by the mixing rule, $\sigma_{i j}=\left(\sigma_{i i}+\sigma_{j j}\right) / 2$ and $\epsilon_{i j}=\sqrt{\epsilon_{i i} \epsilon_{j j}}$.

The Monte Carlo simulation is as follows. (i) Place water molecules in a spherical container. (ii) Move, rotate the water molecules, and exchange water molecules in the cluster with monomers in the vapor. To reduce the center-ofmass motion of the cluster, one water molecule is kept at a fixed position. For this molecule, only rotational move is taken. The volume of the spherical container $v$ is chosen large enough so that the cluster properties are not affected by the wall of the container. Following the previous study, the radius of the spherical container is taken to be $15 \AA^{20,19,21}$ With this radius, the frequency of the water molecules hitting the wall was found to be extremely small, that is to say, the volume occupied by the cluster is much smaller than the volume of the container. The carrier-gas particles are placed in a rectangular cell which encloses the spherical container. Only the translational move is attempted for carrier-gas particles. The periodic boundary condition is imposed if any carrier-gas molecule moves outside the cell. Note that any translational move of a water molecule would be rejected if the molecule moved outside the spherical container.

In the simulation, $P_{i}$ is evaluated from the ratio of the number of Monte Carlo steps of the occurrence of the $i$-mer in the container to the total number of Monte Carlo steps. To carry out the simulation more efficiently, we divided the interval $\left[1, i_{\max }\right]$ into a number of smaller intervals. $P_{i}$ is then computed within one small interval at a time. Eventually, we patch the results of $P_{i}$ in each interval together to obtain the complete $P_{i}$ versus $i$ curve. In each interval, we terminate the Monte Carlo simulation after 100000 cycles equilibration if the least frequent cluster has been sampled 10000 times. For those intervals in which the cluster size is less than 10 , the probability distribution is a steep function of $i$. In these cases we terminated the simulation after the least frequent cluster was sampled a few thousand times. Note that in each interval, the sampling was recorded every 100 Monte Carlo cycles.

\section{RESULTS AND DISCUSSION}

Figure 1 shows $\Delta G_{i}$ curves for the carrier-gas pressure $p=0$ and 40 bar. The temperature of the system is fixed at $T=298.15 \mathrm{~K}$. The supersaturated-vapor density of water is given by $\Omega z=0.12 \times 10^{-5} \AA^{-3}$ where $z=e^{\mu_{1} k_{B} T} / \gamma_{T} \gamma_{R}$. We

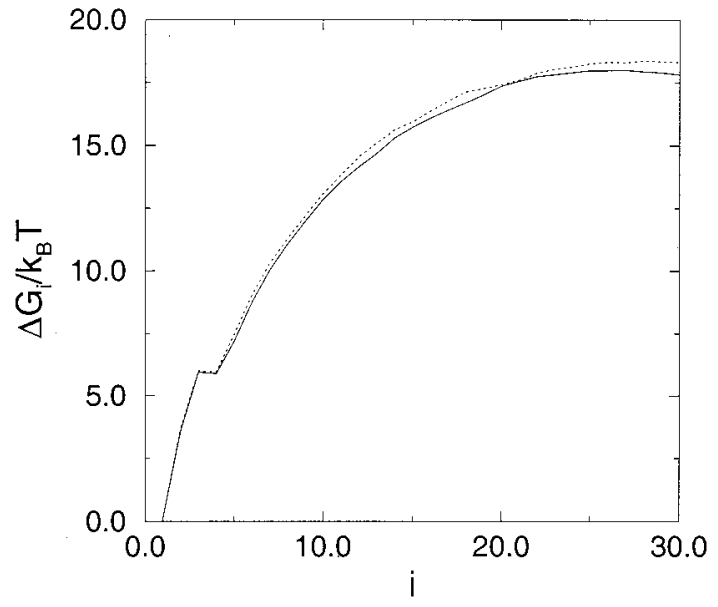

FIG. 1. The free energy of formation of water cluster $\Delta G_{i} / k_{B} T$ at carriergas pressure $0 \mathrm{bar}$ (solid line) and $40 \mathrm{bar}$ (dotted line). The supersaturated vapor is at the temperature $298.15 \mathrm{~K}$ and density $\Omega z=0.12 \times 10^{-5} \AA^{-3}$.

found the nucleation barrier at 40 bar carrier-gas pressure is only slightly higher than that at 0 bar. In fact, their difference is hard to see. The conclusion is that the effect of carrier-gas pressure on the barrier height to nucleation is indeed negligible when the system temperature is high. Experimental evidence has already demonstrated that the carrier-gas effect is more pronounced at relatively low temperatures. ${ }^{6}$ To confirm the experimental finding, we carried out Monte Carlo simulation for the system at a lower temperature, i.e., $T=240 \mathrm{~K}$. Figure 2 shows the $\Delta G_{i}$ curves at carrier-gas pressure $p=0$ and 60 bar. We set the supersaturated-vapor density of water, $\Omega z=0.2$ $\times 10^{-7} \AA^{-3}$. The size of critical cluster at these conditions is about 30 , which is nearly the same as that when the system is at the higher temperature $298.15 \mathrm{~K}$. Clearly, one can see that the barrier height of nucleation is appreciably higher for the higher carrier-gas pressure (60 bar). The barrier-height enhancement amounts to about $2.5 k_{B} T$.

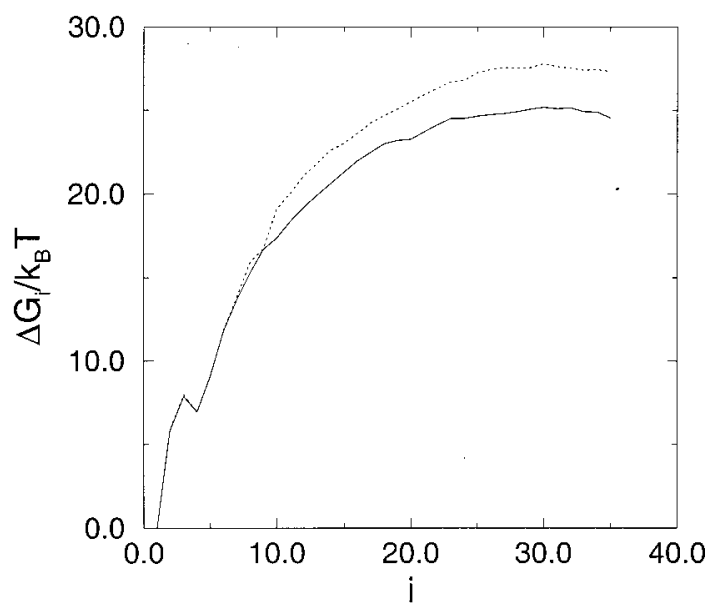

FIG. 2. The free energy of formation of water cluster $\Delta G_{i} / k_{B} T$ at the carrier-gas pressure 0 bar (solid line) and 60 bar (dotted line). The supersaturated vapor is at the temperature $240 \mathrm{~K}$ and density $\Omega z=0.2$ $\times 10^{-7} \AA^{-3}$. 


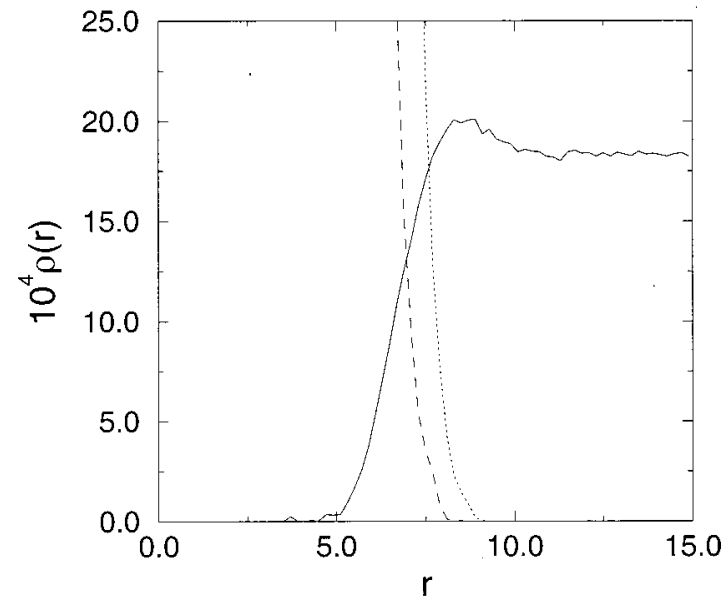

FIG. 3. $\rho(r)$ of the carrier gas (solid line) inside a water cluster of size 30 . Density profiles of $\mathrm{O}$ (dashed line) and $\mathrm{H}$ (dotted line) are also shown to locate the surface of the water cluster. The carrier-gas pressure is $60 \mathrm{bar}$ and the temperature is $240 \mathrm{~K} . r$ is in unit of $\AA$ and $\rho$ is in unit of $\AA^{-3}$.

We examined whether our simulation results are consistent with the nucleation theorem for binary nucleation. ${ }^{24}$ According to that theorem, the derivative of the nucleation barrier $W^{*}$ with respect to the chemical potential $\mu_{k}$ of the component $k$ at fixed temperature is directly related to the size the critical cluster, that is,

$$
\left(\frac{\partial W^{*}}{\partial \mu_{k}}\right)_{T}=-\Delta n_{k}^{*}
$$

where $\Delta n_{k}^{*}$ is the excess number of molecules of component $k$ in the critical cluster over that in the vapor. If $\Delta n_{k}^{*}$ is known, one can easily determine whether the nucleation barrier increases or decreases with the chemical potential (or pressure) of the carrier gas. Let $\Delta n_{c}^{*}$ denote $\Delta n_{k}^{*}$ for the carrier-gas component. It can be evaluated via

$$
\Delta n_{c}^{*}=\int_{v} \rho(r) 4 \pi r d r-\rho_{v} v,
$$

where $v$ is the excluded volume of water cluster, $\rho_{v}$ is the vapor density of the carrier gas, and $\rho(r)$ is the density profile of the carrier gas molecules inside the water cluster. Specifically, in the Monte Carlo simulation, $\rho(r)$ is determined via

$$
\rho(r)=\langle N(r)\rangle / \Delta V,
$$

where $\Delta V=4 \pi / 3\left[(r+\Delta r)^{3}-r^{3}\right]$ is the volume of a spherical bin with thickness $\Delta r$ and $N(r)$ is the number of carriergas particles in the bin. Note that $r$ is measured from the center of mass of the water cluster. Thus, $\Delta n_{c}^{*}(r)$ can be calculated numerically via

$$
\Delta n_{c}^{*}(r)=\sum \rho(r) \Delta V-\rho \sum \Delta V,
$$

where $\Sigma$ denotes the sum over those spherical bins up to the distance $r$.

Figure 3 shows $\rho(r)$ of the carrier-gas particles inside the water cluster of size 30 at carrier-gas pressure $60 \mathrm{bar}$ and temperature $240 \mathrm{~K}$. To guide the eye, we also plotted density

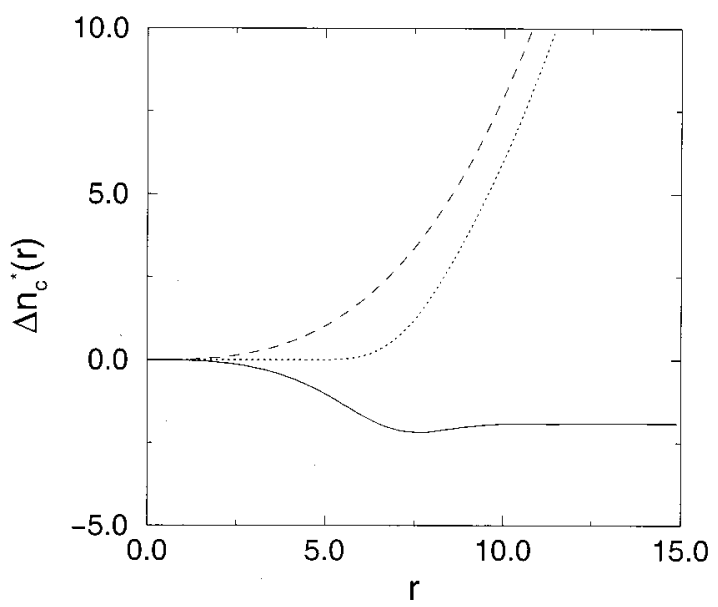

FIG. 4. $\Delta n_{c}^{*}(r)$ (solid line) calculated from the density profile of carrier gas shown in Fig. 3. The dotted and the dashed lines represent data for the first and second term in Eq. (16), respectively. $r$ is in unit of $\AA$.

profiles of oxygen and hydrogen sites near the surface of the water cluster. We used $\Delta r=0.2 \AA$. One can see in Fig. 3 that a hump is shown in the $\rho(r)$ curve, indicating that some carrier-gas molecules are adsorbed at the surface of water cluster. This behavior is also seen in the density profile of planar surface, evaluated from the density functional theory, ${ }^{12}$ of a simple Lennard-Jones fluid in the presence of a carrier gas. This surface adsorption may lead to a positive value of $\Delta n_{c}^{*}$, but sometimes may not. Figure 4 shows $\Delta n_{c}^{*}(r)$ calculated from the density profile of carrier gas shown in Fig. 3. $\rho_{v}$ is estimated from the value of $\rho(r)$ at the region where it levels off (at about $r=12 \AA$ ). At about $r$ $=7 \AA, \Delta n_{c}^{*}(r)$ shows a minimum and it starts to increase with $r$ because of the surface adsorption. Eventually, $\Delta n_{c}^{*}(r)$ becomes flat near $r=12 \AA . \Delta n_{c}^{*}$ is estimated from the value of $\Delta n_{c}^{*}(r)$ at the flat region, which gives $\Delta n_{c}^{*} \sim-2$. According to the nucleation theorem [Eq. (13)], a negative $\Delta n_{c}^{*}(r)$ indicates that the barrier height of nucleation should increase with the carrier-gas pressure, which is consistent with the result shown in Fig. 2.

Figures 5 and 6 show the same curves as those in Figs. 3 and 4, but the system is at a higher temperature, $298.15 \mathrm{~K}$, and a carrier-gas pressure of 40 bar. In this case, we found $\Delta n_{c}^{*} \sim-1$. Again, our finding is consistent with the nucleation theorem that the barrier-height change at the higher temperature (see Fig. 1) is less than that at the lower temperature (see Fig. 2).

Generally, the surface adsorption of the carrier-gas molecules onto the surface of the cluster will result in a reduction ${ }^{25,26}$ of surface tension. In fact, this reduction alone would give rise to a smaller barrier height of nucleation, opposite to our results. However, this apparent contradiction can be resolved by using an argument of Luijten et al.,, ,12,13 who suggested that the carrier-gas effect in water /nitrogen system can be viewed as a result of two competing factors: the enhancement of equilibrium vapor pressure which lowers the supersaturation, and the reduction of surface tension due to the surface adsorption of carrier-gas molecules. Our results therefore suggest that the increased barrier height of 


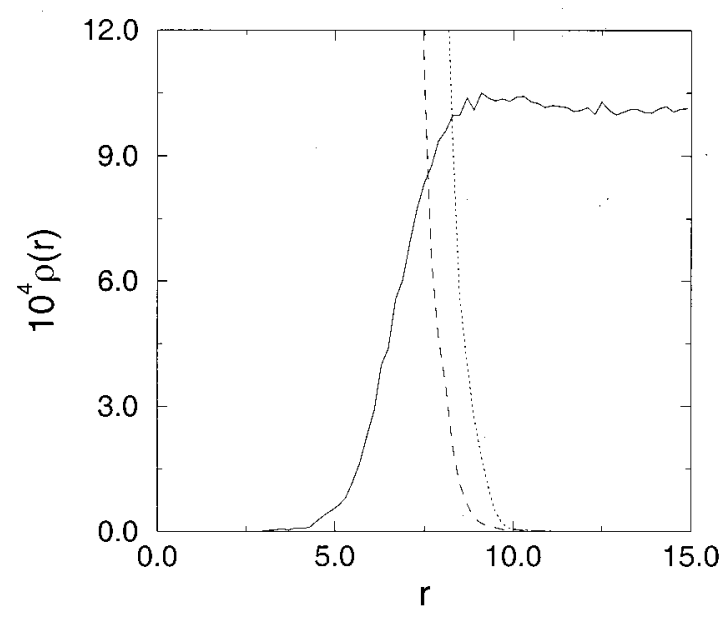

FIG. 5. $\rho(r)$ of the carrier gas (solid line) inside a water cluster of size 30. Density profiles of $\mathrm{O}$ (dashed line) and $\mathrm{H}$ (dotted line) are also shown to locate the surface of the water cluster. The carrier-gas pressure is $40 \mathrm{bar}$ and the temperature is $298.15 \mathrm{~K} . r$ is in unit of $\AA$ and $\rho$ is in unit of $\AA^{-3}$.

nucleation at the higher carrier-gas pressure stems from the decrease of supersaturation.

Kashchiev $^{17}$ recently proposed a simple theory to explain the effect of carrier-gas pressure on nucleation. He found that the carrier pressure can either stimulate or inhibit the nucleation process, depending upon the sign of the effective molecular volume

$$
b=2 b_{12}-b_{22}-v_{0},
$$

where $b_{12}$ and $b_{22}$ are, respectively, the second mixed virial coefficient of the target and carrier gases and the second virial coefficient of the carrier gas, and $v_{0}$ is the molecular volume of the target gas. If $b<0$, the supersaturation (or the chemical potential difference, $\Delta \mu$ ) will be reduced compared to that in the absence of the carrier gas. If $|b|$ is on the order of $10^{-27} \mathrm{~m}^{3}$, the theory predicts that the carrier-gas pressure should be on the order of tens or hundreds of atmospheric pressure to yield appreciable effect. This theoretical predic-

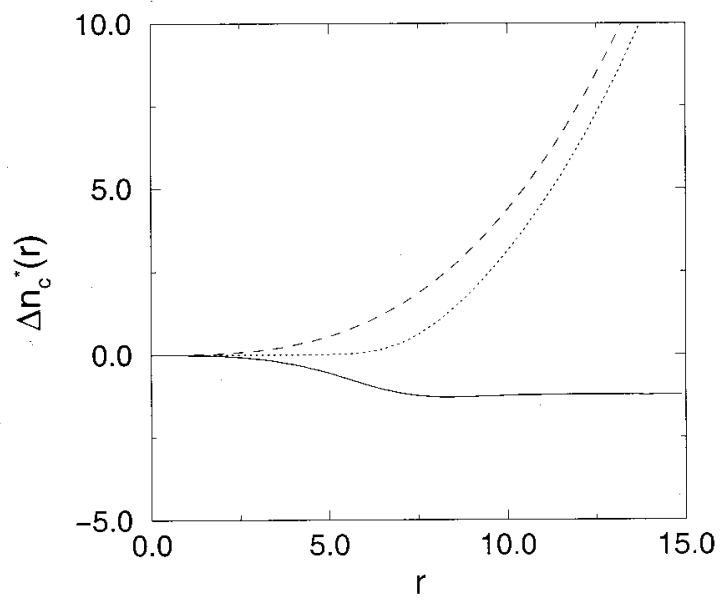

FIG. 6. $\Delta n_{c}^{*}(r)$ (solid line) calculated from the density profile of carrier gas shown in Fig. 5. The dotted and the dashed lines represent data for the first and second term in Eq. (16), respectively. $r$ is in unit of $\AA$. tion appears to be consistent with our simulation results. Furthermore, the effective molecular volume $b$ should be negative in the case of water/nitrogen system at $T=240 \mathrm{~K}$. At a higher temperature, $298.15 \mathrm{~K}$, it seems that $|b|$ becomes much smaller than $10^{-27} \mathrm{~m}^{3}$, based on Eq. (17) and the simulation results. Thus, the carrier-gas effect on the barrier to nucleation is negligible even at 40 bar of carrier gas.

\section{CONCLUSION}

We have carried out Monte Carlo simulation to compute the free energy of formation of water clusters in the presence of nitrogen carrier gas. We find that there are no appreciable changes in the nucleation barrier as the carrier-gas pressure increases from 0 to 40 bar when the system is at the higher temperature, $298.15 \mathrm{~K}$. At the lower temperature, $240 \mathrm{~K}$, however, we find the barrier height of nucleation increases appreciably as the carrier-gas pressure increases from 0 to 60 bar.

Our simulation results are consistent with the binarynucleation theorem which states that the excess number of the carrier-gas molecules relative to the background, $\Delta n_{c}^{*}$, is simply the first derivative of the nucleation barrier with respect to the carrier-gas chemical potential. From the Monte Carlo simulation of the critical cluster, we found $\Delta n_{c}^{*}$ is negative. This means that the nucleation barrier will increase with the carrier-gas pressure.

We also find that the density profile of the carrier gas shows evidence of surface adsorption, which generally results in a reduction of surface tension. The latter factor alone would lead to a lower barrier height to nucleation, opposite to our finding. However, this apparent contradiction can be resolved by taking into account another competing factor-a reduction of the supersaturation due to the presence of the carrier gas. This factor seems to play a more important role in the observed carrier-gas effect in water/nitrogen system.

\section{ACKNOWLEDGMENTS}

We are grateful for valuable discussions with Professor Heist and Professor van Dongen. This work is supported by the National Science Foundation.

${ }^{1}$ J. L. Katz, J. A. Fisk, and V. M. Chakarov, in Nucleation and Atmospheric Aerosols, edited by N. Fukuta and P. E. Wagner (Deepak, Hampton, 1992).

${ }^{2}$ R. H. Heist, M. Janjua, and J. Ahmed, J. Phys. Chem. 98, 4443 (1994).

${ }^{3}$ R. H. Heist, J. Ahmed, and M. Janjua, J. Phys. Chem. 99, 375 (1995).

${ }^{4}$ A. Bertelsmann, R. Stuczynski, and R. H. Heist, J. Phys. Chem. 100, 9762 (1996).

${ }^{5}$ D. Kane and M. S. El-Shall, J. Chem. Phys. 105, 7617 (1996).

${ }^{6}$ C. C. M. Luijten, K. J. Bosschaart, and M. E. H. van Dongen, J. Chem. Phys. 106, 8116 (1997).

${ }^{7}$ J. A. Fisk and J. L. Katz, J. Chem. Phys. 104, 8649 (1996).

${ }^{8}$ D. Kane, S. P. Fisenko, and M. S. El-Shall, Chem. Phys. Lett. 102, 6846 (1995)

${ }^{9}$ O. V. Vasilev and H. Reiss, J. Chem. Phys. 105, 2946 (1996).

${ }^{10}$ O. V. Vasilev and H. Reiss, Phys. Rev. E 54, 3950 (1996).

${ }^{11}$ V. M. Novikov, O. V. Vasilev, and H. Reiss, Phys. Rev. E 55, 5743 (1997).

${ }^{12}$ C. C. M. Luijten and M. E. H. van Dongen, J. Chem. Phys. 111, 8524 (1999).

${ }^{13}$ C. C. M. Luijten, P. Peeters, and M. E. H. van Dongen, J. Chem. Phys. 111, 8535 (1999).

${ }^{14}$ D. W. Oxtoby and A. Laaksonen, J. Chem. Phys. 102, 6846 (1995).

${ }^{15}$ See I. J. Ford, in Ref. 1. 
${ }^{16}$ I. J. Ford, J. Aerosol Sci. 23, 447 (1992).

${ }^{17}$ D. Kashchiev, J. Chem. Phys. 104, 8671 (1996).

${ }^{18}$ K. J. Oh and X. C. Zeng, J. Chem. Phys. 112, 294 (2000).

${ }^{19}$ K. J. Oh, G. T. Gao, and X. C. Zeng (unpublished).

${ }^{20}$ I. Kusaka, Z.-G. Wang, and J. H. Seinfeld, J. Chem. Phys. 102, 913 (1995).

${ }^{21}$ G. T. Gao, K. J. Oh, and X. C. Zeng, J. Chem. Phys. 110, 2533 (1999).
${ }^{22}$ H. Reiss and R. K. Bowles, J. Chem. Phys. 112, 1390 (2000).

${ }^{23}$ H. J. C. Berendsen, J. R. Grigera, and T. P. Straatsma, J. Phys. Chem. 91, 6269 (1987).

${ }^{24}$ D. W. Oxtoby and D. Kashchiev, J. Chem. Phys. 100, 7665 (1994).

${ }^{25}$ J. W. Gibbs, Collected Works I (Yale University Press, New Haven, CT, 1948).

${ }^{26}$ R. Massoudi and A. D. King, Jr., J. Phys. Chem. 78, 2262 (1974). 